\title{
Trend in Antimicrobial Resistance of Staphylococcus aureus: Results from the China Antimicrobial Surveillance Network (CHINET) in the Last I5-Year- Period Reports (2005-2019)
}

Yingjian Liang ${ }^{1} *$

Lidi Qiu ${ }^{2, *}$

Xiaobin Zheng $\mathbb{D}^{\prime}$

Jing Liu'

'Department of Pulmonary and Critical Care Medicine (PCCM), The Fifth Affiliated Hospital of Sun Yat-sen University, Zhuhai, 519000, People's Republic of China; ${ }^{2}$ Department of Infectious Disease Intensive Care Unit, The Fifth Affiliated Hospital of Sun Yatsen University, Zhuhai, People's Republic of China

*These authors contributed equally to this work
Correspondence: Jing Liu; Xiaobin Zheng Department of Pulmonary and Critical Care Medicine (PCCM), Fifth Affiliated Hospital of Sun Yat-sen University, 52 East Meihua Road, Zhuhai City, 519000,

People's Republic of China

Tel/Fax +867562528733

Email liujing25@mail.sysu.edu.cn;

zhxbin@mail.sysu.edu.cn
Abstract: In this report, we analyze the th as in an crobial istance of Staphylococcus aureus according to the last 15-year-p reports of $\mathrm{C}$ antimicrobial Surveillance Network (CHINET). It is encourag g tha ere is a deceasing MRSA proportion in the infection of adults as well as bettor activity of no beta-lactam agents in vitro in recent years. However, childhood MRSA afection as well as t. tigecycline-resistant MRSA isolate in China deserves increasing oncern.

Keywords: Staphylococ aureus, timicrobial resistance, China Antimicrobial Surveillance Netyork

St nyloco us aur s (S. aureus) is a notorious opportunistic-pathogen responsifor a section in a variety of infectious diseases, some of which are letha Antibiotic resistance surveillance can provide effective information in the clinical rapy of $S$. aureus infection for physicians. The consecutive microbioloical surverllance among clinically significant isolates (such as $S$. aureus) has been on ing in mainland China since 2005, named the China Antimicrobial Surveillance Network (CHINET). More and more hospitals from various provinces in China have participated in this programme since 2005. In the 2019 report, there were 36 tertiary hospitals from over 20 provinces or autonomous regions. Moreover, antimicrobial resistance of over 100,000 no-duplicate $S$. aureus isolates from routine laboratory work were submitted until 2019, which were informative to clinical physicians in treatment. Herein, we deciphered a trend of antimicrobial resistance among $S$. aureus from CHINET according to the last 15-year-period (2005-2019) reports, for physicians to develop a strategy in optimizing their approach of $S$. aureus infections.

All the data was downloaded from the website of the China Antimicrobial Surveillance Network (http://www.chinets.com). In vitro antimicrobial susceptibility of $S$. aureus isolates were determined by modified Kirby-Bauer disk diffusion method according to the Clinical and Laboratory Standards Institute (CLSI) guidelines. S. aureus ATCC 25923 was used as a quality control. Tigecycline (TGC) was evaluated according to the US FDA standard. 
A total of 132,284 non-duplicate clinical isolates of $S$. aureus (including methicillin-sensitive $S$. aureus, MSSA and methicillin-resistant $S$. aureus, MRSA) was submitted during the 15 -year surveillance period, $54,438(41.15 \%)$ of which were MRSA, according to CHINET. The overall MRSA rate decreased from $69 \%$ in 2005 to $31.4 \%$ in 2019 , and the prevalence of MRSA strains in adult cases had a similar trend that peaked in $2005(85.8 \%)$ and then declined over the following 15 years $(2019,31.7 \%)$. The MRSA rate in children's infection cases has grown several times, peaking at $33.4 \%$ (2014), and has been stable at about $30 \%$ in the last 4 -year period. Since 2012, the proportion of MSSA have been overweight than MRSA on CHINET. Regarding antimicrobial resistance, erythromycin (ERY) exhibited poor activity in MRSA (resistance rate over $70 \%$ since 2005) as well as MSSA (resistance rate over $45 \%$ since 2005) in this series of reports. Clindamycin (CLI) also exhibited low-to-moderate antimicrobial susceptibility in both MRSA (resistance rate over $50 \%$ since 2005) and MSSA (resistance rate over $20 \%$ since 2005). There is an abrupt growth in gentamicin (GEN) resistant MRSA isolates in 2006 (from 36.3\% to $89.3 \%$ ), but GEN has shown increased activity in vitro against MRSA since then. Levofloxacin (LEV) presented an extremely high resistance rate fr 005 to 2012 (over $80 \%$ ). Antimicrobial actiy of MR in GEN, LEV, and rifampicin (RIF) ho gradually mproved since 2012. Son on the tested antimicrobials reveale a hig" res res ratio in MRSA isolate rather an ISSA of the same time

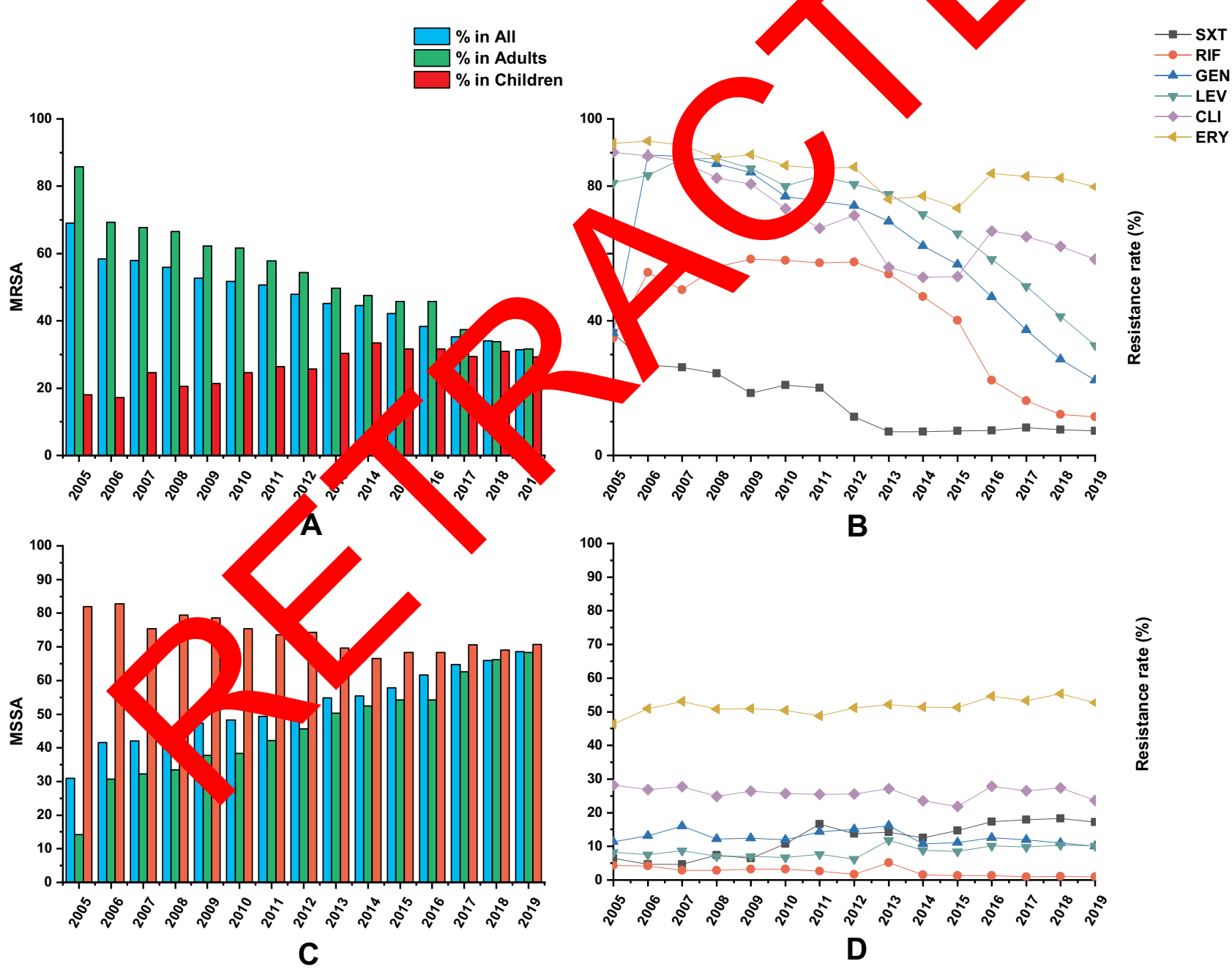

Figure I Trend in Antimicrobial Resistance of MRSA as well as MSSA in last I5-year-period CHINET reports (2005-2019). (A and C) The prevalence of MRSA and MSSA in adults and children, respectively. (B and D) The non-beta-lactam antibiotic agent resistance of MRSA and MSSA, respectively.

Notes: TGC resistant rate was firstly reported in 2019 , which is $0.7 \%$ in MRSA and $0.1 \%$ in MSSA (not shown in figure). Only tertiary hospitals data was included in 2019. Abbreviations: ERY, erythromycin; CLI, clindamycin; GEN, gentamicin; LEV, levofloxacin; RIF, rifampicin; SXT, trimethoprim-sulfamethoxazole. 
point, including RIF, GEN, LEV, CLI, and ERY, but trimethoprim-sulfamethoxazole (SXT) was not. Notably, it was the first time that CHINET reported TGC resistant MRSA on 2019 (0.7\%). All S. aureus isolates were found to be susceptible to vancomycin, teicoplanin, and linezolid (Figure 1).

The CHINET Antimicrobial Surveillance Program has been ongoing since its inception in 2005 and the trends in MRSA proportion have declined in the last 15-year report, which is consistent with other regional as well as national surveillance programs, such as SENTRY. ${ }^{2}$ The noteworthy decreasing of MRSA isolate in the infection of adults is encouraging, but there has been concern about the considerable proportion in children, as it has greatly enhanced from $17.2 \%$ (2005) to $29.3 \%$ (2019), peaking at $33.4 \%$ in 2014 . Moreover, it has stably contributed to about $30 \%$ of $S$. aureus infection in recent years, which indicated increased focus on infection prevention and stronger control interventions on children should be taken.

It is also encouraging that most of the non-beta-lactam agents exhibited better and better activity in vitro in recent years, both in MRSA and MSSA, but there is a high resistance rate of ERY, suggesting its potential failure of $S$. aureus infection. There is no surveil nce about the TGC susceptibility of $S$. aureus CHI report until 2019. However, our pre ous a 1 othe work has revealed the low TGC resis nce rat $f \mathrm{MRS} A$ before 2019. ${ }^{3,4}$ Perhaps detailed $y$ earch "GC resistant MRSA should be under perfo d as there scanty data about its molecular related ss, res tant pattern, and virulence genes profiling in hina. In sun ary, we elucidated the trend of antimi obial among $S$. aureus based on the last 15-year-perio $\mathrm{CH}^{\mathrm{ET}}$ report. Perhaps further insight about ildho MRSA Aection as well as TGC resistant RSA plates ana should be warranted in the fu

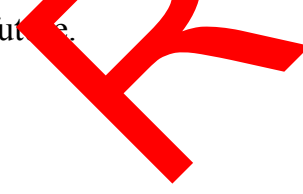

Infection and Drug Resistance

\section{Publish your work in this journal}

Infection and Drug Resistance is an international, peer-reviewed openaccess journal that focuses on the optimal treatment of infection (bacterial, fungal and viral) and the development and institution of preventive strategies to minimize the development and spread of resistance. The journal is specifically concerned with the epidemiology of

\section{Limitations}

All the data was from CHINET, which indicated that those data of the hospital beyond this program may be ignored.

\section{Data Sharing Statement}

The data that supported this study was based on available data.

\section{Acknowledgment}

We gratefully acknowledge the contributions of the members of CHINET for collection of alates tested in the last 15 years (http://y W.chin .com/Data/ AntibioticDrugFast).

\section{Funding}

This work was por a by Apen project of Key Laboratory Tropic Disea Control (Sun Yat-sen Universit, Ministry a ducation (grant number 2020kfkt07).

\section{pisclosure}

The authors port no conflicts of interest in this work.

\section{Returences}

1. nbers HF, Deleo FR. Waves of resistance: Staphylococcus aureus in the antibiotic era. Nat Rev Microbiol. 2009;7(9):629-641. doi:10.1038/nrmicro2200

2. Diekema DJ, Pfaller MA, Shortridge D, Zervos M, Jones RN. Twentyyear trends in antimicrobial susceptibilities among Staphylococcus aureus from the SENTRY antimicrobial surveillance program. Open Forum Infect Dis. 2019;6(Suppl 1):S47-S53. doi:10.1093/ofid/ofy270

3. Liang Y, Tu C, Tan C, et al. Antimicrobial resistance, virulence genes profiling and molecular relatedness of methicillin-resistant Staphylococcus aureus strains isolated from hospitalized patients in Guangdong Province, China. Infect Drug Resist. 2019;12:447-459. doi:10.2147/IDR.S192611

4. Wu D, Wang Z, Wang H, et al. Predominance of ST5-II-t311 clone among healthcare-associated methicillin-resistant Staphylococcus aureus isolates recovered from Zhejiang, China. Int $J$ Infect Dis. 2018;71:107-112. doi:10.1016/j.ijid.2018.04.798 antibiotic resistance and the mechanisms of resistance development and diffusion in both hospitals and the community. The manuscript management system is completely online and includes a very quick and fair peerreview system, which is all easy to use. Visit http://www.dovepress.com/ testimonials.php to read real quotes from published authors. 